\title{
SGLT2 Inhibitors: Alternatives or Complementary Therapeutic Options Alkhatib $\mathrm{J}^{1,2^{*}}$
}

${ }^{1}$ Department of Legal Medicine, Toxicology of Forensic Science and Toxicology, School of Medicine, Jordan University of Science and Technology, Jordan

${ }^{2}$ International Mariinskaya Academy, department of medicine and critical care, department of philosophy, Academician secretary of department of Sociology

Corresponding Author: Ahed J Alkhatib

Address: Department of Legal Medicine, Toxicology of Forensic Science and Toxicology, School of Medicine, Jordan University of Science and Technology, Jordan; Tel: oog62795905145; E-mail: ahed.alkhatib64@yahoo.com, ajalkhatib@just.edu.jo,drahedalkhatib@yahoo.com

Received date: 26 January 2020; Accepted date: 01 February 2020; Published date: 08 February 2020

Citation: Alkhatib J. SGLT2 Inhibitors: Alternatives or Complementary Therapeutic Options. Diab Res Open Access. 2020 Feb 08;2(S1):15-16.

Copyright (C) 2020 Alkhatib J. This is an open-access article distributed under the Creative Commons Attribution License, which permits unrestricted use, distribution, and reproduction in any medium, provided the original work is properly cited.

\section{Keywords \\ SGLT2 Inhibitors; Diabetes; Pancreas}

Diabetes type 2 is a real challenge of health across the world. Therapeutic options are varied and include various lines such as starting with giving metformin with increasing doses and ending with insulin at increasing dose model.

I think that a science philosophical problem has been involved in the research and science of diabetes. The definition of diabetes depends mainly on determining insulin level or function. But insulin level is not measured as a direct cause of diabetes, while the glucose level is measured as a result of diabetes (indirect proof). The perceived idea of diabetic cause is insulin deficiency as either in concentration or function. A functional insufficiency of the pancreas was the focus point of view which requires giving insulin treatment as diabetes progresses.

We have recently shown that insulin treatment policies need to be revised because the insulin level increases with diabetic progression. According to this context, we can understand how diabetes is continuously developing. Conventional treatments of diabetes need to be reviewed so that the level of glucose decreases without increasing the level of insulin.

There is a big question that needs to be clarified in relation to sugar metabolism. At the average, an individual intakes amount of sugar more than reflected by monitoring of glucose level. Accordingly, there is something hidden in diabetes and needs to be more clarified.

The introduction of SGLT2 Inhibitors has come to inhibit the reabsorption of glucose by kidneys. It is expected to offer benefits for the patients through decreasing glucose levels without increasing insulin levels, which is extremely an important option.

Another benefit of SGLT2 inhibitors is its potential use as a prophylactic option through reducing weight 
Citation: Alkhatib J. SGLT2 Inhibitors: Alternatives or Complementary Therapeutic Options. Diab Res Open Access. 2020 Feb 08;2(S1):15-16.

which participates in preventing the development of obesity and diabetes. 